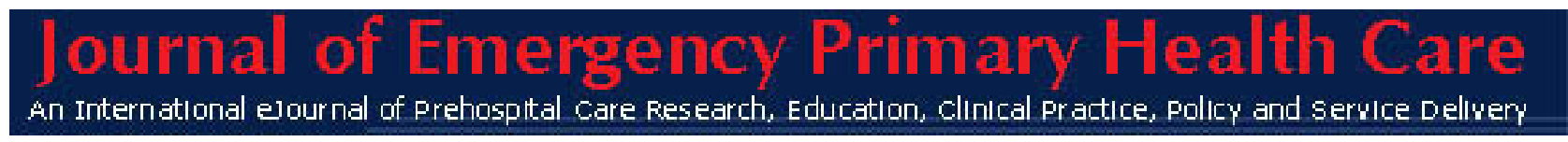

1447-4999

\title{
EDITORIAL
}

\section{PROPOSED “COCHRANE PREHOSPITAL CARE FIELD”}

Article No. 990063

Frank Archer and Erin Smith

A process to establish a Prehospital Care Field within the Cochrane Collaboration is currently underway.

There is increasing emphasis internationally to base policy and practice decisions on evidence. This includes prehospital and emergency primary health care. The Cochrane Collaboration is an international organisation that aims to help people make well informed decisions about health care by preparing, maintaining and promoting the accessibility of systematic reviews of the effects of health care interventions. It is a not for profit organisation established as a company, limited by guarantee, and registered as a charity in the United Kingdom.

The Cochrane Collaboration was formed just over 10 years ago and has rapidly gained an enhanced international status of developing and promoting one source of evidence base. Two interesting events on the Australian scene emphasise the significance of the Cochrane Collaboration and its work. First, the Australian Government recently announced that it would make access to the Cochrane Library free to everyone in Australia. Secondly, systematic reviews published either in the Cochrane Library or other journals earn the same academic status as other publications published in peer reviewed journals, thereby allowing academics and universities to include Cochrane style systematic reviews to be included in their research output.

The Cochrane Collaboration does not contend that all health care interventions need be justified by randomised control trials or a Cochrane systematic review. This is a misconception that may have discouraged involvement of prehospital care researchers and users of research information from the Cochrane Collaboration. What the Cochrane Collaboration does claim is that many interventions do lend themselves to a methodology of a systematic review and in these cases this should be the most comprehensive review of the available evidence as possible. The Cochrane Collaboration recognises the value of other forms of evidence but has chosen to focus as its core business, the methodologies which involve randomised controlled trials and systematic reviews. The NH\&MRC (National Health and Medical Research Council) guidelines for quality identify these methodologies as the highest form of quality in considering healthcare evidence.

At present there are 50 Collaborative Review Groups within the Cochrane Collaboration of which 25 could be seen to relate to prehospital care. At present there are 39 systematic reviews and 6 protocols which relate to prehospital care listed in the Cochrane Library. However, there is no specific prehospital care focus within the Cochrane Collaboration to assist in developing these systematic reviews.

To establish a new entity within the Cochrane Collaboration requires attainment of a number of steps in an approval process. 


\section{Journal of Emergency Primary Health Care (JEPHC), Vol.1, Issue3-4, 2003}

The concept of a possible Cochrane Prehospital Care Field was raised by a number of individuals as far back as 1996/97. However, it was only in mid 2002 that the first step of an informal international meeting was held. This meeting was held in association with the $9^{\text {th }}$ International Conference on Emergency Medicine held in Edinburgh, Scotland when 19 participants from 11 countries held an informal meeting to discuss the merits and values of seeking to establish a Cochrane Prehospital Care Field. The meeting unanimously supported progressing with this objective. Subsequent informal meetings were held at AMBEX 2002 in Harrogate (UK) and at an International EMS Conference in Dubai (UAE) in October 2003.

The second step in the process was approved at a formal international meeting which was held in Melbourne on the $7^{\text {th }}$ May 2003 as a pre-Congress workshop in association with the $13^{\text {th }}$ World Congress on Disaster and Emergency Medicine. This international meeting was hosted by the Australasian Cochrane Centre and was a meeting to explore the possibility of establishing a Cochrane Prehospital Care Field.

During this one day workshop, attended by 30 participants from 9 countries, the principles, objectives, structure and function of the Cochrane Collaboration were outlined, and the role and functions of a Field in the Cochrane Collaboration were explored.

National and International speakers focussed on the relationship, the evolving evidence base in prehospital care, and included:

- A/Prof. Michael Sayre, Principle Investigator, National (USA) EMS Research Agenda project on behalf of the National Association of EMS Physicians

- A/Prof. Ian Jacobs, Director of Western Australian Prehospital Care Research Unit and Chairman of the Australian Resuscitation Council

- Dr Andrew Marsden, Consultant Medical Director, Scottish Ambulance Service and board member of the Faculty of Prehospital Care, the Royal College of Surgeons of Edinburgh

- Mr Mal Boyle, Monash University Centre for Ambulance \& Paramedic Studies and the Australian Prehospital Research Directory

- Mr Steve McDonald, Australasian Cochrane Centre

- A/Prof. Michael Abramson, Cochrane Airways Group

- Dr Liz Waters, Cochrane Health Promotion and Public Health Field

- A/Prof. Frank Archer, Director, Monash University Centre for Ambulance and Paramedic Studies

These speakers were supported by consumer representatives from the Epilepsy Foundation of Victoria and the Asthma Foundation of Victoria.

The meeting endorsed the following resolution: "That amongst a range of strategies to improve the international evidence base of prehospital care, this formal exploratory international meeting believes that there are mutual benefits to both the Cochrane Collaboration and international prehospital care and seeks to register a Cochrane Prehospital Care Field within the Cochrane Collaboration”.

This could be seen as a significant step for developing the international evidence base for Prehospital care.

An international steering committee with representatives from four countries has progressed the resolution in Melbourne and developed a scope of activity for the proposed Cochrane Prehospital Care Field for formal endorsement by the Cochrane Collaboration. Individuals interested in being kept informed of the progress of this proposal, or who would like to contribute to the development of the scope document, should register their interest at erin.smith@med.monash.edu.au . Comments on 
the draft scope document received before 24th May 2004 will be reviewed by the Steering Committee for inclusion in the revised scope document to be submitted to the Cochrane Representative Committee in June, when it is hoped the new entity will be formally registered.

The Steering Committee will be leading an international workshop and a Field meeting at the 12th Cochrane Colloquium to be held in Ottawa, Canada 2-6 October, 2004. Further information about the Colloquium is available at http://www.colloquium.info/.

\section{Key References:}

The Cochrane Collaboration homepage is http://www.cochrane.de/.

The sites below contain further information on the various entities within the Collaboration, and these can be explored to identify those which relate to prehospital care.

General information on the Collaboration: http://www.cochrane.de/cochrane/general.htm

Overview of Cochrane review groups and centres: http://www.cochrane.de/cochrane/crgs.htm

Abstracts of Cochrane reviews: http://www.cochrane.de/cochrane/revabstr/mainindex.htm 